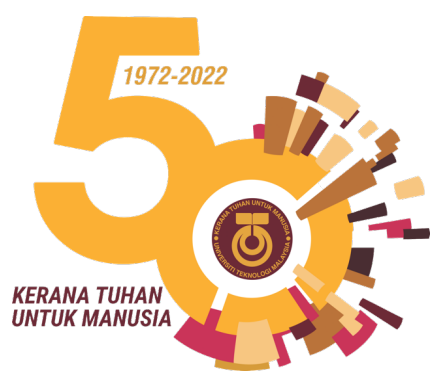

\title{
Exponential Growth Model and Stochastic Population Models: A Comparison via Goat Population Data
}

\author{
Nurul Ashikin Mohamad Radzia, Haliza Abd. Rahman ${ }^{\mathrm{a}, \star}$, Shariffah Suhaila \\ Syed Jamaludina, Arifah Bahar ${ }^{\mathrm{b}}$
}

a Department of Mathematical Sciences, Faculty of Science, Universiti Teknologi

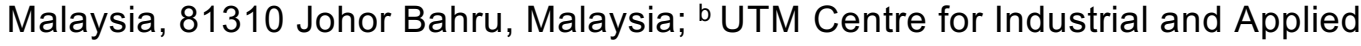
Mathematics (UTM-CIAM), 81310 Johor Bahru, Malaysia

Abstract A population dynamic model explains the changes of a population in the near future, given its current status and the environmental conditions that the population is exposed to. In modelling a population dynamic, deterministic model and stochastic models are used to describe and predict the observed population. For modelling population size, deterministic model may provide sufficient biological understanding about the system, but if the population numbers become small, then a stochastic model is necessary with certain conditions. In this study, both types of models such as exponential, discrete-time Markov chain (DTMC), continuous-time Markov chain (CTMC) and stochastic differential equation (SDE) are applied to goat population data of small size. Results from the simulations of stochastic realizations as well as deterministic counterparts are shown and tested by root mean square error (RMSE). The SDE model gives the smallest RMSE value which indicate the best model in fitting the data.

Keywords: Population dynamics, exponential model, discrete-time Markov chain, continuous-time Markov chain, stochastic differential equation.

*For correspondence: halizarahman@utm.my

Received: 6 Sep 2021

Accepted: 18 Feb 2022

(C) Copyright Radzi et al. This article is distributed under the terms of the Creative Commons Attribution License, which permits unrestricted use and redistribution provided that the original author and source are credited.

\section{Introduction}

A stochastic process is a probability process where any process in nature can be analyzed in terms of probability. It is a random process evolving in time where if full knowledge of a system and its entire past is known, its value at future times is uncertain [1]. A stochastic process is an outcome of an experiment which are all functions of time. In other words, stochastic process assigns a sample function which is the time function to each outcome.

The use of stochastic model had been increased dramatically over the past decades due to recent interest in experimental methods such as population dynamic. Experiments such as laser traps and fluorescent protein that can be measured in a single cell shows that the dynamic of essential species behave in naturally stochastic manner [1]. The mathematical implication of this observation is that standard deterministic models for dynamics needs to sometimes be replaced with corresponding stochastic model.

A deterministic process is a process whereby its future is completely determined by its present and its past states. However, for modelling population size, if it is large then deterministic model may provide sufficient biological understanding about the system, but at any time the population numbers do become small, then a stochastic model is necessary with certain conditions [2]. Three simulations of birth-death model which generated from the same process may result in exponential growth, linear growth or die out 
completely. The only differences are the purely random effect. If plots of small simulation size reasonably close to the deterministic curve the deterministic approach is sufficient otherwise a stochastic description needs to be developed.

Generally defined, population is a group of individuals of the same species living together in a particular place. Berryman [3] defines population as "a group of individuals of the same species that live together in an area of sufficient size to permit normal dispersal and or migration behavior and in which numerical changes are largely determined by birth and death processes (BDPs)".

To describe population growth, it is essential to know the processes that cause changes in population size through time. The simplest model for population growth is exponential model where there is no predator or competitor in the population, resources is unlimited, and the environment is constant. This is a deterministic model suitable for modelling close population growth with density-independent factors.

Malthus in his book "An Essay on the Principle of Population" which first published anonymously in 1798 and later rewritten to the $6^{\text {th }}$ version in 1826 argued if population grew without restrain, its growth would be controlled by the means of survival. He proposed a population model that has geometric pattern growth for 'unchecked' population growth and arithmetic progression for 'checked' population growth. Velhulst in 1838 formulated logistic model of population growth which takes a sigmoidal modal followed by a decrease in growth and bound by a carrying capacity due to environmental pressures. Velhurst's model and its variation perhaps the most well-known model classic population model [4].

Markov in 1907 began to study chance process where in this process, the outcome of a given experiment can affect the outcome of the next experiment which is called a Markov chain. According to Jeppson [5] the need for stochastic models in population dynamics was recognized in the late 60's. Deterministic approaches involving ordinary differential equations which approximate large number of discrete populations however these approaches proven less useful when applied to small sample sizes. Thus, to address this issue stochastic population models such as continuous-time Markov Chain (CTMC) models are often used when dealing with low number of populations [6]. Allen and Allen [7] presented a work on the similarities between three stochastic population models namely discrete-time Markov Chain (DTMC), continuous-time Markov Chain (CTMC) and stochastic differential equation (SDE) models, with regard persistence time. Specifically, these models are applied to estimate persistence times for population satisfying logistic growth. The relations between the mean persistence time and higher-order moments are compared. The results showed that the dynamics of these three logistic models are in close agreement even for a small initial population size. Thus, this study aims to apply deterministic model and the three stochastic population models in real animal population data with small population size.

\section{Methodology}

\section{Data and sources}

The purpose of this study is to find the best fitted model to the goat population data. The population of domestic goat Capra Hircus which are known as a source of milk, meat and fibre is obtained from 16 March 2014 to 15 March 2015 from a company in Batu Pahat named Ladang Sunnah. It consists of the number of birth and death daily and the total population of goat.

\section{Methodology framework}

The data will be fitted to both type of models exponential model which is deterministic model, CTMC, DTMC and SDE models which are stochastic models. The models are then compared using the Root Mean Squared error (RMSE) whereby the model with the least RMSE is considered as the best fitted model.

\section{Stochastic Processes}

A stochastic process $(X(t): t \in T C, X(t) \in S)$ is a collection of random variables that are indexed by a set $T$ and it takes values in a set $S$. The variable $t$ usually represent time. For a discrete-time setting, the notation is $T=\{0,1,2,3, \ldots\}$ or $T=[0, \infty)$ for a continuous-time setting. The set $S$ is called the state space 
and this set may be countable (discrete) or non-countable (continuous). This study is particularly interested in stochastic processes that satisfy the Markov Property.

A Markov process $X(t)$ is a process such that the evolution in the future depends on its past only through the present value. Let $t_{0}$ be the present time, the future i.e $\left\{X(t) ; t_{0}<\mathrm{t}\right\}$ depends on its past $\left\{X(s) ; s \leq t_{0}\right\}$ only through the present value $X\left(t_{0}\right)$. This property is named after a Russian mathematician Markov for his famous work on these processes and it is called the Markov property. To define the property; consider a set of $n$ arbitrarily chosen instants in time, denoted as $t_{1}<t_{2}<\cdots<t_{n-1}<t_{n}$. Then, $X(t)$ is called a Markov process if

$$
P\left[\left(X\left(t_{n}\right) \leq x \mid x\left(t_{i}\right) ; i=1,2, \ldots, n-1\right)\right]=P\left[X\left(t_{n}\right) \leq x \mid x\left(t_{n-1}\right)\right]
$$

Markov processes can be discrete-time or continuous-time. $\left\{X_{n}: n=0,1,2, \ldots\right\}$ is a Markov process where often called as a Markov sequence. More precisely, $\left\{X_{n}\right\}$ is called a Markov chain when $X_{n}$ takes a finite or countably infinite number of states $S$. The term DTMC usually means a simple Markov Chain is defined. The term Markov chain is often defined largely to include continuous-time processes. Thus, a discrete-state, continuous-time Markov process is called CTMC and for the case where the number of states $S$ is continuous, the process is called stochastic differential equation (SDE).

\section{Birth-Death Processes}

The simplest type of population growth is exponential. Exponential population growth models are influenced by birth and deaths since the measure of change in population size per unit time and the intrinsic rate of increase are both per-capita measures of an individual's contribution to population growth. These two parameters are constant and influenced by birth and deaths in the population. The birth and death rates stay the same through time, regardless of population size which is called densityindependent.

According to Renshaw [2], a simple birth death process (BDP) is developed under the assumptions that the probabilities of an organism to reproduce or die remain constant. It means that the individual birth and death rates remain the same. The probabilities are also independent of population size. That means all individuals of the populations are assumed capable of reproducing. It is either the organism is asexual or if it is bisexual, only females are considered having the capability. Also, the shortages of males are assumed never occurred.

This can only be true if intraspecific competitions do not occur. However, in a closed environment, the growth of expanding population must be limited by shortage of resources sooner or later. After that, there is a level when the demands made on these resources prevent further growth and later cause the population to be at its saturation level, a value determined by the 'carrying capacity' of the environment. This is called a general BDP.

Different type of interference occurs between individual organisms competing for available resources. From Renshaw [2], in the midst of wild competition, the total number of individuals $(N)$ in a fixed region can change for only four reasons, namely
i. $\quad$ Birth
- rate depends on $N$;
ii. Death $(N>0)$
- rate depends on $N$;
iii. Immigration
- rate is independent on $N$;
iv. Emigration $(\mathrm{N}>0)$
- rate is independent on $N$.

According to Allen [8] in the basic population model, birth and death are denoted as $\lambda(i)$ and $\mu(i)$ respectively. These rates are assumed to be continuous and differentiable functions of the population size $i$. In addition, it is assumed that there are numbers $K$ and $N$ such that $0<K<N$ and 
i. $\lambda(0)=\mu(0)=0$ and $\lambda(i)=0$ for $i \geq N$;

ii. $\lambda(i)>0$ for $i \in(0, N)$ and $\mu(i)>0$ for $i>(0, N]$;

iii. $\lambda(i)>\mu(i)$ for $i \in(0, K)$;

iv. $\lambda(i)=\mu(i)$ for $i \in(K, N)$.

\section{Deterministic Birth-Death Model}

Three different stochastic models mentioned in Section 2.1 are described based on the above assumptions. But prior to modeling the data with stochastic model, a deterministic birth-death model which is a basic birth death model will be firstly considered. By considering both types of models, the adequacy of the type of model either deterministic or stochastic, will be justified suitable for small population data.

Considering the classic deterministic approach let $N(t)$ denotes the random variable for total population size at time $t$. Then in the subsequence small time interval of length $\Delta t$, an increase in population size is due to single organism is $\lambda \Delta t$. Thus, an increase in size due to all $N(t)$ organisms is $\lambda \Delta t N(t)$. Same goes with a decrease in population size due to a single organisms which is $\mu \Delta t$, the decrease in size due to all organisms is $\mu \Delta t N(t)$. Thus,

$$
\begin{array}{r}
N(t+\Delta t)=N(t)+\lambda \Delta t N(t)-\mu \Delta t N(t) \\
=N(t)+(\lambda-\mu) \Delta t N(t) \\
\frac{N(t+\Delta t)-N(t)}{\Delta t}=(\lambda-\mu) N(t)
\end{array}
$$

By letting $\Delta t \rightarrow 0$, the above equation becomes the differential equation,

$$
\frac{d N(t)}{d t}=(\lambda-\mu) N(t)
$$

with solution $N(t)=N(0) e^{(\lambda-\mu) t}$. The net rate of increase $(\lambda-\mu)$ may be either positive or negative. The population size is predicted at time $t$ with absolute certainty once the initial population size $N(0)$ and the net rate of increase is known. For the deterministic model, exponential population growth model is chosen compared to logistic population growth model since its properties correspond with simple BDP. It means the intrinsic rate is influenced by birth and death which are constant parameters in BDP.

\section{Stochastic Birth-Death Model}

The three types of stochastic models will be formulated by defining relationships among the random variables assuming the Markov property holds. Let $N(t)$ denotes the random variable for the total population size at time $t$. Assume birth and death rates satisfy the four assumptions mentioned earlier with in all cases, $N$ represents the maximum population size.

\section{DTMC Model}

Let $N(t)$ denote the discrete random variable for the number of individuals at time $t$ with associated probability function $P[N(t)=i]=p_{i}(t)$ where $i=0,1,2, \ldots, N$. The probability distribution is $p(t)=$ $\left(p_{0}(t), p_{1}(t), \ldots, p_{N}(t)\right)^{T}$ for $t \in\{0, \Delta t, 2 \Delta t, \ldots\}$ and $\Delta t$ be a fixed time interval. It is assumed that $\Delta t$ is sufficiently small such that the possible changes are at most increase or decrease by one in the time interval $(t, t+\Delta t)$, either a birth or a death. A birth occurs with probability $\lambda(i) \Delta t$ and a death occurs with probability $\mu(i) \Delta t$. Denote the transition probabilities as $p_{j i}(\Delta t)=P[N(t+\Delta t)=j \mid N(t)=i]$ where

$$
p_{j i}(\Delta t)=\left\{\begin{array}{cl}
\lambda(i) \Delta t & ; j=i+1 \\
\mu(i) \Delta t & ; j=i-1 \\
1-[\lambda(i) \Delta t+\mu(i) \Delta t] & ; j=i \\
0 & ; \quad \text { otherwise }
\end{array}\right.
$$

Then, $p_{i}(t+\Delta t)$ satisfies the following equations where, $p_{i}(t+\Delta t)$ is the probability of a population size is $i$ at time $t+\Delta t$. Thus the probability distribution associated with the population size over time is found by repeated multiplication of the transition matrix. 
For $i=1, \ldots, N-1$,

$$
p_{i}(t+\Delta t)=\lambda(i-1) \Delta t p_{i-1}(t)+\mu(i+1) \Delta t p_{i+1}(t)+[1-[\lambda(i) \Delta t+\mu(i) \Delta t]] p_{i}(t)
$$

For $i=0$,

$$
p_{0}(t+\Delta t)=\mu(1) \Delta t p_{1}(t)+p_{0}(t)
$$

For $i=N$,

$$
p_{N}(t+\Delta t)=\lambda(N-1) \Delta t p_{N-1}(t)+[1-\mu(N)] \Delta t p_{N}(t)
$$

Equation (2) can also be expressed in matrix form as

$$
p(t+\Delta t)=\boldsymbol{P}(\Delta t) p(t)
$$

where matrix $\boldsymbol{P}(\Delta t)=p_{i j}(\Delta t)$ is the transition matrix. To ensure matrix $\boldsymbol{P}$ is stochastic matrix in which the entries are nonnegative and the column elements sum to one, it is assumed that $\max _{i}\{[\lambda(i)+\mu(i)] \Delta t\} \leq 1$.

\section{CTMC Model}

A CTMC birth death process (BDP) is a process where the time is continuous but the random variable for the number of individuals at time $t$ is discrete. The discrete random variable $N(t), t \in[0, \infty)$ has an associate probability function $P[N(t)=i]=p_{i}(t)$. The infinitesimal transition probability for small $\Delta t$ is

$$
p_{j i}(\Delta t)=\left\{\begin{array}{cl}
\lambda(i) \Delta t+o(\Delta t) & ; \quad i=j-1, i \in\{1, \ldots, N\} \\
\mu(i) \Delta t+o(\Delta t) & ; j=j+1, i \in\{0, \ldots, N-1\} \\
1-[\lambda(i) \Delta t+\mu(i) \Delta t]+o(\Delta t) & ; \quad i=j, i \in\{1, \ldots, N\} \\
0(\Delta t) & ; \text { otherwise }
\end{array}\right.
$$

where $o(\Delta t) \rightarrow 0$ as $\Delta t \rightarrow 0$. In the limit $\Delta t \rightarrow 0$, a system of differential equations for the probabilities $P[N(t)=i]=p_{i}(t)$ can be shown to satisfy the forward Kolmogorov differential equations.

For $i \geq 1$,

$$
\frac{d p_{i}(t)}{d t}=\lambda(i-1) \Delta t p_{i-1}(t)+\mu(i+1) \Delta t p_{i+1}(t)-[\lambda(i)+\mu(i)] p_{i}(t)
$$

For $i=0$,

$$
\frac{d p_{0}(t)}{d t}=\mu(1) p_{1}(t)
$$

The DTMC transition matrix and CTMC differential equations are closely related when $\Delta t$ is small. In the DTMC model equation (2) which the Kolmogorov differential equation is obtained as,

$$
\begin{gathered}
\frac{p(t+\Delta t)-p(t)}{\Delta t}=\frac{\boldsymbol{P}(\Delta t)-\boldsymbol{I}}{\Delta t} p(t) \\
\frac{d p}{d t}=\boldsymbol{Q} p
\end{gathered}
$$

where

$$
Q=\lim _{\Delta t \rightarrow 0} \frac{\boldsymbol{P}(\Delta t)-\boldsymbol{I}}{\Delta t}
$$

The system difference equation like the equation (3) may be considered a discrete-time approximation to the differential system in equation (4)[7]. 
Because of the Markov property; the inter-event time in CTMC model has an exponential distribution which has the memoryless property. The inter-event time is the waiting time until an event occurs or the time between successive events. Let $N(t)=n$ and $T_{n}$ denote inter-event time, the continuous random variable for the time to the next event. Then, the sum of all probabilities of all possible events where there is a change in state, either birth or death

$$
\sum_{j=0, j \neq n}^{\infty} p_{j n}(\Delta t)=a(n) \Delta t+o(\Delta t) .
$$

Then, the probability of no change in state is

$$
p_{n n}(\Delta t)=1-a(n) \Delta t+o(\Delta t)
$$

Let $G_{n}(t)$ be the probability that the process remains in its current state for a time of length $t$. Then $G_{n}(t)$ can be expressed in terms of the inter-event time $T_{n}$.

$$
G_{n}(t)=P\left[T_{n}>t\right]
$$

For $\Delta t$ sufficiently small,

$$
G_{n}(t+\Delta t)=G_{n}(t) p_{n n}(\Delta t)=G_{n n}(t)(1-a(n) \Delta t+o(\Delta t))
$$

Subtract $G_{n}(t)$ from both sides of equation (5) and divide by $\Delta t$. The taking the limit as $\Delta t \rightarrow 0$, it follows that

$$
\frac{d G_{n}(t)}{d t}=-a(n) G_{n}(t)
$$

Equation (6) is the first order differential equation and homogeneous with initial condition $G_{n}(0)=1$. The solution is

$$
G_{n}(t)=P\left[T_{n}>t\right]=e^{-a(n) t}
$$

Thus, the probability that $T_{n} \leq t$ is

$$
P\left[T_{n} \leq t\right]=1-G_{n}(t)=1-e^{-a(n) t}=F_{n}(t)
$$

The function $F_{n}(t)$ is the cumulative distribution function for the inter-event time $T_{n}$. It corresponds to an exponential random variable with parameter $a(n)$. The random variable $T_{n}$ can be expressed in terms of the distribution function $F_{n}(t)$ and uniform random variable $U$. This relationship is very useful when stochastic realization is numerically simulated.

$$
\begin{gathered}
F_{n}(t)=1-e^{-a(n) t} \\
P\left[F_{n}^{-1}(U) \leq t\right]=P\left[F_{n}\left(F_{n}^{-1}(U)\right) \leq F_{n}(t)\right]=P\left[U \leq F_{n}(t)\right]=F_{n}(t)
\end{gathered}
$$

Thus, the inter-event time $T_{n}$, given $N(t)=n$ satisfies

$$
T_{n}=F_{n}^{-1}(U)=-\frac{\ln (1-U)}{a(n)}=-\frac{\ln (U)}{a(n)}
$$

where $a(n)=(\lambda-\mu)(n)$. The next event is either a birth or death. A birth occurs with probability $\frac{\lambda}{\lambda+\mu}$ 
and death with probability $\frac{\mu}{\lambda+\mu}$.

\section{SDE Model}

For this formulation, let the time $t \in[0, \infty)$ and the state space $N(t) \in[0, N]$. Let $p(x, t)$ denotes the probability density function for the continuous random variable $N(t)$.

$$
P[N(t) \in[a, b]]=\int_{a}^{b} p(x, t) d x
$$

The birth rates and death rates are assumed to satisfy the four conditions stated previously. The probability distribution as $\Delta t \rightarrow 0$ satisfies the forward Kolmogorov differential equation.

$$
\frac{\partial(x, t)}{\partial t}=-\frac{\partial([\lambda(x)-\mu(x) p(x, t)])}{\partial x}+\frac{1}{2} \frac{\partial^{2}([\lambda(x)-\mu(x) p(x, t)])}{\partial x^{2}}
$$

for $x \in(0, N), t \in(0, \infty)$ and $p(x, 0)=\delta\left(x-x_{0}\right)$, where $\lambda(x)-\mu(x)$ and $\lambda(x)+\mu(x)$ are the infinitesimal mean and infinitesimal variance respectively. From equation (7), sample paths $N(t)$ of the stochastic process satisfy the following equation,

$$
N(t)=n_{0}+\int_{0}^{t}[\lambda N(u)-\mu N(u)] d u+\int_{0}^{t} \sqrt{\lambda N(u)+\mu N(u)} d B(u)
$$

In equation (8), $B$ is the standard Brownian motion, where $\Delta B(t)=B(t+\Delta t)-B(t)$ has a normal distribution, $N(0, \Delta t)$. The first integral in equation (8) is Riemann integral while the second integral is an Ito stochastic integral. For notational convenience, equation (8) is often expressed as the stochastic differential equation,

$$
\frac{d N(t)}{d t}=\lambda N(t)-\mu N(t)+\sqrt{\lambda N(t)+\mu N(t)} \frac{d B(t)}{d t} N(0)=n_{0}>0
$$

It is noticed in equation (9) the deterministic term is identical to the right hand-side of the deterministic equation in (1). The stochastic term in (9) is due to the variations in birth and death rates.

\section{Root Mean Squared Error}

The performance of the four models in this study is measured using the root mean squared error (RMSE). RMSE is the mean squared difference between the estimated values and the observed values.

$$
\text { RMSE }=\sqrt{\frac{1}{n} \sum_{i=1}^{n}\left(Y_{i}-\hat{Y}_{i}\right)^{2}}
$$

\section{Results and discussion}

The initial number of goat population is 10 when the data is recorded and the final population size is 42 . The goats died naturally or had been slaughtered. Figure 1 shows the goat population from day 1 until 365 days later. 


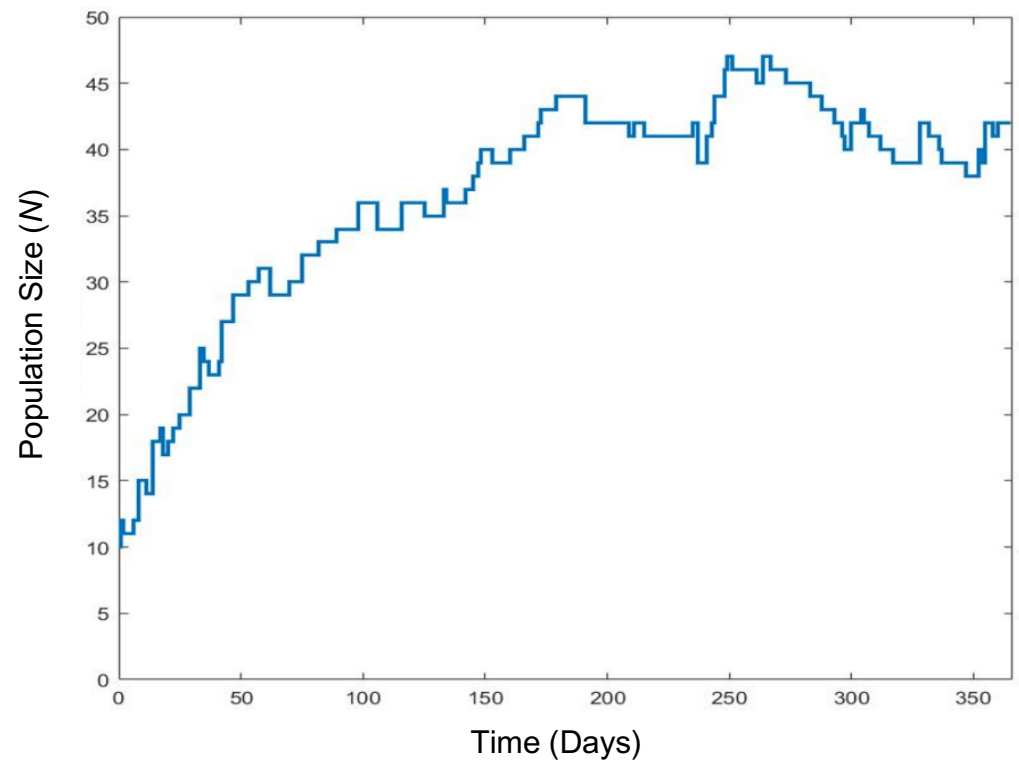

Figure 1. Goat populations from 16 March 2014 until 15 March 2015

\section{Parameter estimation}

The deterministic equation (1) has a parameter called net rate of increase $(\lambda-\mu)$. The net increase may be either positive or negative. For populations to grow naturally in the exponential manner, the number of individuals must be small enough relative to the available food resource to guarantee that there is no competition between individuals [2].

Based on the goat population, the initial population size, $N(0)$ is 10 while the population size after one year $N(1)$ is 42 . Thus, by inserting these values into the solution of (1), an estimate $(\lambda-\mu)$ of the net annual growth rate can be obtained.

$$
\begin{gathered}
N(t)=N(0) e^{(\lambda-\mu) t} \\
42=10 e^{(\lambda-\mu)(1)} \\
\lambda-\mu=\ln \left(\frac{42}{10}\right)=1.4351 \\
\text { Birth rate, } \lambda=\frac{\text { The number of birth in 1 year }}{\text { Total population size }}=\frac{75}{42}=1.7857 \\
\text { Death rate, } \mu=\frac{\text { The number of death in 1 year }}{\text { Total population size }}=\frac{43}{42}=1.0238 \\
\text { Net increase }=\text { growth rate }=\lambda-\mu=0.7619
\end{gathered}
$$

From equation (11) it means there are about 1.79 births per individual, per year and from equation (12) there are about 1.02 deaths per individual, per year. As from equation (13), there are about 0.76 new individuals per existing individual, per year. The positive value in the equation implies the goat population is growing instead of dying. Let multiply (13) with existing population size,

$$
0.7619 \times 42=32.00001 \approx 32 .
$$


Thus, in a year the goat population grew by 32 individuals at a rate of 0.7619 . This type of growth is where a population grown in proportions to its size is called exponential growth. It means the bigger the population size, the more rapidly it grows. Next, the estimated parameters are used to simulate stochastic realizations using MATLAB R2011b.

\section{Simulations of the sample path of stochastic models.}

The plot of the observed data, deterministic model and stochastic models are shown in Figure 2. For the stochastic models, simulation of the sample path for each model are generated and plotted in Figure 2. In the figure, the line represented SDE simulation indicates a close approximation of the actual data where both graphs seem eventually overlap. This portrays the more accurate and close approximation of SDE model from the actual data of goat population in the long run compared to the other stochastic models.

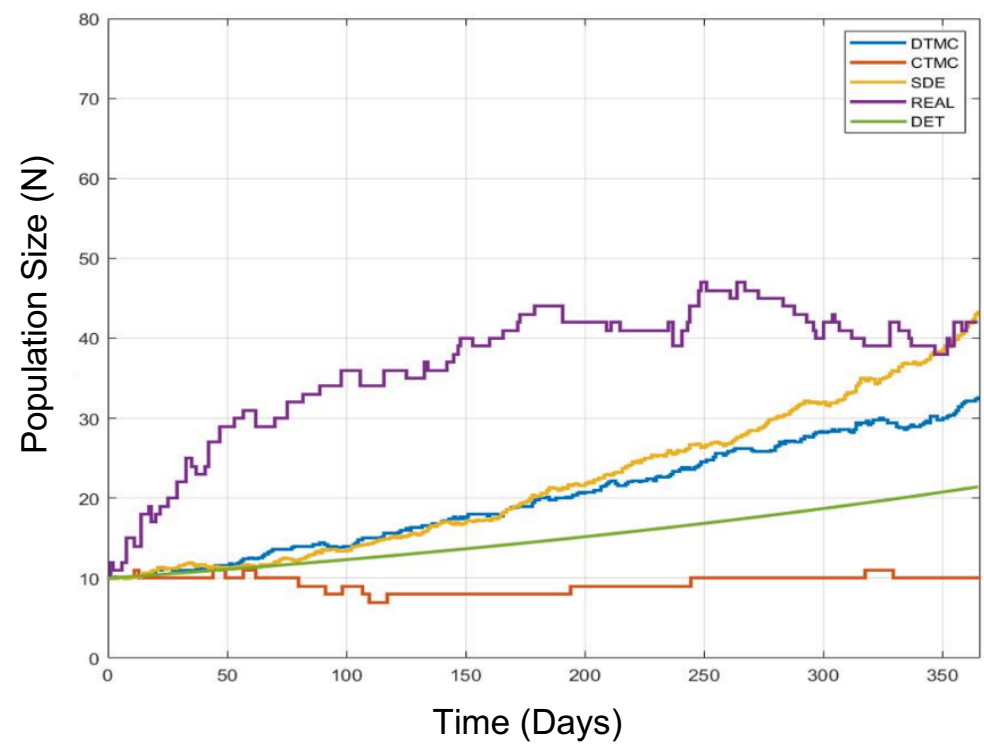

Figure 2. Comparison of goat population data with deterministic, DTMC, CTMC and SDE models

The summary of the results in terms of the calculated RMSE for each model are provided in Table 1. Table 1 shows the RMSE for deterministic models, DTMC, CTMC and SDE. It can be seen that SDE model has the smallest value compared to the other models. From Figure 2 it shows that the graph of SDE model is the closest to the goat population data. These provide the justification that SDE is the best model to fit the goat population data.

Table 1. RMSE for the deterministic, DTMC, CTMC and SDE models

\begin{tabular}{cc}
\hline Model & RMSE \\
\hline Deterministic & 22.5095 \\
DTMC & 17.4198 \\
CTMC & 23.5034 \\
SDE & 15.7900 \\
\hline
\end{tabular}

As a conclusion for this set of data, stochastic model with continuous-time parameter such as SDE is adequate for modeling birth death process since it has the most minimum value of RMSE indicating the sample paths of SDE provide the closest approximation to the real data. 


\section{Conclusions}

Deterministic models are regularly used as modeling population size however if the number of population size become small then stochastic model may be necessary in certain conditions. For comparison purposes the implementation of deterministic BDP, stochastic DTMC, CTMC and SDE models had been carried out in this study to find the best fitted model of the goat population data with small population size. The deterministic exponential model is initially fitted to the data and the estimated parameters of the deterministic population growth model are then employed in the simulations of the three stochastic models. Simulations of the three stochastic models as well as its deterministic counterpart were shown and compared by calculating the RMSE for each models. Results show that SDE model has the smallest RMSE and provide adequate fit for the goat population data. This indicates the more accurate and closer approximation of SDE model from the actual data compared to the other stochastic models and considered as the best model to describe the goat population data.

\section{Conflicts of interest}

The authors declares that there is no conflict of interest regarding the publication of this paper.

\section{Funding statement}

This work was financially supported by Research University Grant with vote no: Q.J130000.2526.08H60.

\section{Acknowledgments}

The author would like to express their greatest appreciation to Universiti Teknologi Malaysia for providing the Research University Grant with vote no: Q.J130000.2526.08H60.

\section{References}

[1] Anderson, D.F. 2013. Introduction to Stochastic Processes with Applications in the Biosciences, University of Wisconsin at Madison.

[2] Renshaw, E. 1993. Modelling Biological Population in Space and Time, Cambridge University Press.

[3] Berryman, A.A. 2002. Population: A Central Concept for Ecology?, Oikos, 97(3):439-442.

[4] Buckley, F.M. 2010. Analytical Methods for Stochastic Discrete-time Metapopulation Models, The University of Queensland.

[5] Jeppson, T. 2009. Stochasticity in the Extinction Process, Department of Ecology, SLU.

[6] Banks, H.T., Broido, A., Canter, B., Gayvert, K., Hu, S., Joyner, M. \& Link, K. 2012. Simulation algorithms for continuous time Markov chain models, Studies in Applied Electromagnetics and Mechanics, 37:3-18.

[7] Allen, L. J. S. \& Allen, E. J. 2003. A comparison of three different stochastic population models with regard to persistence time," Theoretical Population Biology, 64(4):439-449.

[8] Allen, L. J. S. 2010. An Introduction to Stochastic Processes with Applications to Biology, CRC Press. 